\title{
INERTIAL FORCES IN YARN
}

\author{
PRACEK, S.
}

Abstract: We derive the system of coupled nonlinear differential equations that govern the motion of yarn in general. The equations are written in a (non-uniformly) rotating observation frame and are thus appropriate for description of over-end unwinding of yarn from stationary packages. We comment on physical significance of inertial forces that appear in a non-inertial frame and we devote particular attention to a lesser known force, that only appears in non-uniformly rotating frames. We show that this force should be taken into account when the unwinding point is near the edges of the package, when the quasi-stationary approximation is not valid because the angular velocity is changing with time. The additional force has an influence on the yarn dynamics in this transient regime where the movement of yarn becomes complex and can lead to yarn slipping and even breaking.

Key words: inertial forces, dynamics of yarn, balloon theory, non-inertial systems
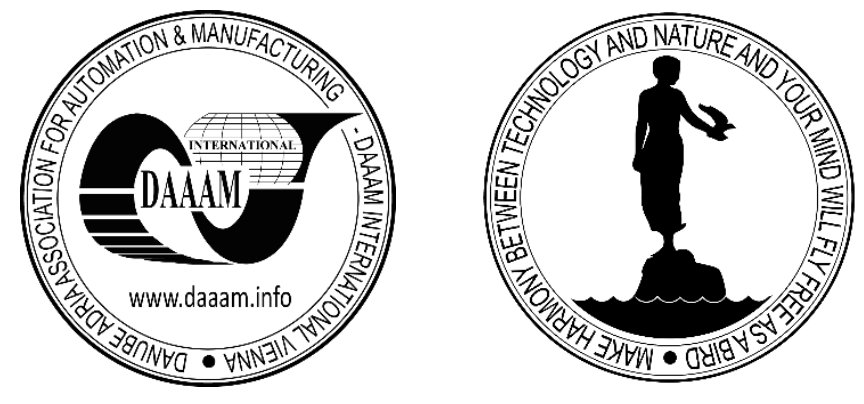

Authors' data: Assoc.Prof. Pracek, S[tanislav]*, * University of ljubljana, NTF, Department of TGO, Snezniska 5, 1000 Ljubljana, Slovenija, stane.pracek@ntf.unilj.si,

This Publication has to be referred as: Pracek, S[tanislav] (2019). Inertial Forces in Yarn, Chapter 08 in DAAAM International Scientific Book 2019, pp.099-108, B. Katalinic (Ed.), Published by DAAAM International, ISBN 978-3-902734-24-2, ISSN 1726-9687, Vienna, Austria

DOI: 10.2507/daaam.scibook.2019.08 


\section{Introduction}

In production of garments thread exists in a sewing process. In order to achive low and constantt tension of thread or yarn it is necessary to optimize the process of unwinding. Computer simulation are now in use for this purpose, so it is important to obtain a mathematical description of yarn motion (Barr\&Catling, 1976; Clark et al.,1998; Padfield, 1956; Mack, 1953; Kothari\&Leaf, 1979; Fraser ,1992; Fraser et al.,1992; Praček, 2002).

The unwinding will be discussed from a theoretical and practical point of view. In this paper we show how inertial forces enter the equation of motion for yarn in a rotating reference frame. In addition to the Coriolis and the centrifugal force, there is another inertial force in rotating frames with the dependent angular velocity. This force affects the yarn dynamics.

\section{Non-inertial reference frames and the fictitious forces}

The Newton's second law $\mathbf{F}=$ ma, where $\mathbf{F}$ is the sum of all forces acting on the body, $\mathrm{m}$ the mass of the body, and $\mathbf{a}$ its acceleration, takes this form only in inertial reference frames. Inertial references frames are all those reference frames which are not accelerating (Giancoli ,2009). In such frames a free body $(\mathbf{F}=0)$ moves with a velocity which is constant both in its direction and its size, since the acceleration of the body is equal to zero. This statement is also know as the law of inertial motion (Giancoli ,2009).

If we introduce another reference frame which moves with a constant velocity $\mathbf{V}$ with respect to the inertial reference frame (so that $\mathbf{x}^{\prime}=\mathbf{x}+\mathbf{V} t$ ), then the law of free motion will take the same form as in the first frame, since the acceleration is equal to zero also in the second, $\mathbf{a}^{\prime}=0$ : in case of free motion, the velocity is constant. The new reference system is also inertial. Empirically it is known that the laws of motion are the same in both reference systems; indeed, the two systems are fully equivalent as far as all their mechanical properties are concerned. There is thus an infinity of inertial reference frames which move with constant velocities relative to each other. In all of these systems the laws of mechanics are exactly the same (Giancoli 2009). This is Galileo's principle of relativity, one of the foundations of mechanics.

Now let us consider a reference frame which moves with a relative acceleration $\mathbf{a}_{\text {rel }}$ with respect to a chosen inertial reference frame. In other words, the relation between the acceleration in the inertial frame, $\mathbf{a}$, and the acceleration in the noninertial frame, $\mathbf{a}^{\prime}$, is given by

$$
\mathbf{a}=\mathbf{a}^{\prime}+\mathbf{a}_{\text {rel }}
$$

If this expression is used in the Newton's second law, we obtain $\mathbf{F}=m\left(\mathbf{a}^{\prime}+\mathbf{a}_{\text {rel }}\right)$. The second term on the right-hand side can be shifted to the left, giving

$$
\mathbf{F}-m \mathbf{a}_{\mathrm{rel}}=m \mathbf{a}^{\prime}
$$


This equation can be called the Newton's law for a non-inertial reference frame. It shows that in a non-inertial frame the body is accelerated not only because of real external forces which are applied on it, but also because of the inertial forces $-m \mathbf{a}_{\text {rel }}$. The inertial forces are not real forces but rather a mathematical trick which simplifies the analysis of mechanical systems, since the equation (2) has exactly the same structure as the standard Newton's law $\mathbf{F}=m \mathbf{a}$ : the acceleration times the mass, ma', is equal to the sum of all external forces $\mathbf{F}$ and all inertial forces $-m \mathbf{a}_{\text {rel }}$ that are applied to the body. For this reason, one can make use of all the tools of analytical mechanics if in the equations one replaces the sum of all external forces with the sum of all external and inertial forces.

The inertial forces are also known as virtual, system, or pseudoforces. These names emphasize that these are not real physical forces, but rather effective forces experiences by an observer in an accelerated reference frame due to inertial effects. It must be constantly kept in mind that the inertial forces do not appear in the equations of motion if these are used to describe motion in an inertial reference frame, even if the motion of the body itself is accelerated. Inertial forces only appear in the equations where the equations themselves are formulated in an accelerated reference frame.

There are numerous situations where the use of non-inertial reference frames is more convenient that that of inertial frames, in spite of the addition of the inertial forces. In weather prediction it is, for example, necessary to solve a complex system of differential equations in a reference frame which is fixed to the Earth. Since the Earth is not an inertial reference frame (due to its rotation), it is crucial to take into account the Coriolis and centrifugal forces. Particularly important is the Coriolis force which makes the low-pressure systems spin in the counter-clockwise direction in the Northern hemisphere.

Non-inertial reference frames are also useful in studying the yarn unwinding. It may be used, for example, when working within the quasi-stationary approximation to describe the yarn unwinding from packages where layers have a large number of yarn loops. On the other hand, when the yarn is being unwound from packages with a small number of loops, the angular velocity of the yarn unwinding is not well defined (constant), thus the problem may just as well be described within a fixed inertial reference frame.

\section{Inertial forces in rotating reference frames with non-constant angular velocity}

Let there be an inertial reference frame $K$ and a non-inertial reference frame $K$, which rotates with respect to the frame $K$ with an angular velocity $\omega(t)$. The angular velocity is not necessarily constant, thus we are dealing with angular acceleration and deceleration. We consider the motion of a body in both frames. Let the velocity in the frame $K$ be denoted as $\mathbf{v}$, and the velocity in the frame $K^{\prime}$ as $\mathbf{v}^{\prime}$. Within the time $\delta t$, the body moves in the frame $K$ by $\mathbf{v} \delta t$, which must equal $\mathbf{v}^{\prime} \delta t$ (the displacement of the body in frame $\left.K^{\prime}\right)$ and $(\omega(t) \delta t) \times \mathbf{r}^{\prime}$ (the displacement of the body due to the rotation of frame $\left.K^{\prime}\right)$. We thus obtain the relation between the two velocities 


$$
\mathbf{v}=\mathbf{v}^{\prime}+\omega \times \mathbf{r}^{\prime}
$$

We can also observe that the relation between the time derivative operations with the two coordinate frames may be written as (Goldstein et al., 2002).

$$
\left(\frac{\partial}{\partial t}\right)_{K}=\left(\frac{\partial}{\partial t}\right)_{K}+\omega \times
$$

The operator expression (4) becomes meaningful only when applied to some (vectorial) function $\mathbf{p}$. It is necessary to write $\mathbf{p}$ to the right of each term, obtaining

$$
\left(\frac{\partial \mathbf{p}}{\partial t}\right)_{K}=\left(\frac{\partial \mathbf{p}}{\partial t}\right)_{K}+\boldsymbol{\omega} \times \mathbf{p}
$$

If this rule is applied to the velocity equation (3), we obtain the relation between the accelerations a and a' (Giancoli ,2009; Goldstein et al., 2002):

$$
\begin{aligned}
\mathbf{a} & =\mathbf{a}^{\prime}+\omega \times \mathbf{v}^{\prime}+\left(\dot{\Phi} \times \mathbf{r}^{\prime}+\omega \times \mathbf{v}^{\prime}\right)+\omega \times\left(\omega \times \mathbf{r}^{\prime}\right) \\
& =\mathbf{a}^{\prime}+2 \omega \times \mathbf{v}^{\prime}+\omega \times\left(\omega \times \mathbf{r}^{\prime}\right)+\dot{\Phi} \times \mathbf{r}^{\prime}
\end{aligned}
$$

The last three terms represent the relative acceleration. If this expression is inserted in the Newton's second law for non-inertial reference frames (2), we obtain (Giancoli ,2009; Goldstein et al., 2002)

$$
\mathbf{F}-2 m \boldsymbol{\omega} \times \mathbf{v}^{\prime}-m \omega \times\left(\boldsymbol{\omega} \times \mathbf{r}^{\prime}\right)-m \dot{\omega} \times \mathbf{r}^{\prime}=m \mathbf{a}^{\prime}
$$

The first force on the left-hand side of the equation is the Coriolis force $2 m \boldsymbol{\omega} \times \mathbf{v}^{\prime}$, the second is the centrifugal force- $m \omega \times\left(\boldsymbol{\omega} \times \mathbf{r}^{\prime}\right)$. Most mechanics textbooks only consider the case of constant angular velocity, therefore the third inertial force is less known and sometimes it is neglected in cases where it does play a role. In the accessible literature on the topic of yarn unwinding this force is not mentioned.

Let us consider the variation of the three inertial forces if we are an observer standing on a carousel. The most directly experiences fictional force is the centrifugal force; due to its presence, one has to grab some handle to avoid being ejected from the carousel. The Coriolis force is observed if an object is thrown from the carousel. The object will not flying in a straight line as in an inertial reference frame, but it will be deflected in a direction perpendicular to its velocity.

The third inertial force is experienced if the carousel suddenly comes to a halt. Due to inertial effects, our body will keep moving and we may fall (unless the handle is held sufficiently strongly). For this reason, the third inertial force is not always negligible, but can have sizable effects when the angular velocity suddenly changes. As discussed in the following, this force must be taken into account at the package edges. 


\section{The equation of motion for yarn}

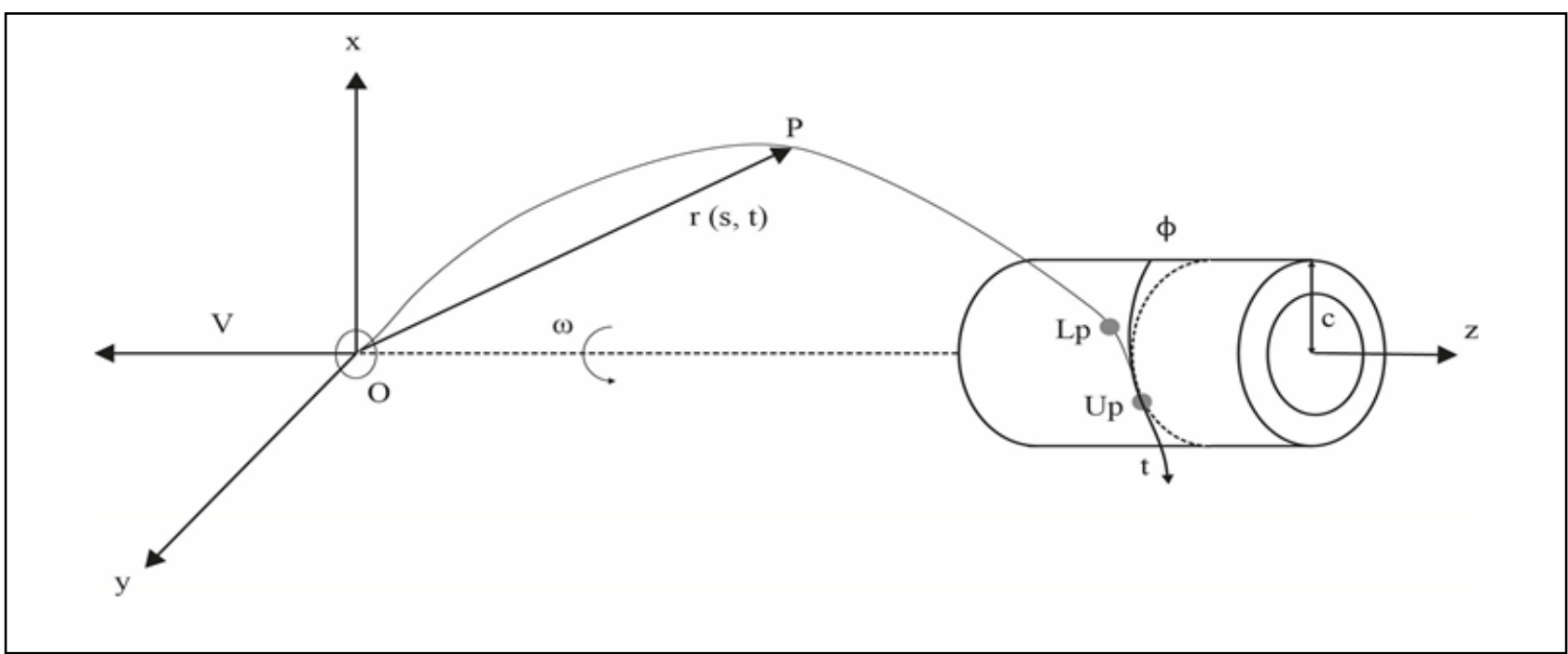

Fig.1. Unwinding yarn from packages.

At the lift-off point Lp the yarn lifts from the package and forms a balloon. At the unwinding point Up the yarn starts to slide on the surface of the package. Angle $\phi$ is the winding angle of the yarn on the package.

The unwinding yarn forms a "balloon" (Fig.1): the yarn rotates with a high angular velocity around the $\mathrm{Z}$ axis and in one turn it defines the contour of a rotational body with one or several balloons. The shape of the rotational body - the balloon, changes only little in one period of the motion. The motion of the curve $\mathbf{r}(s)$ can therefore be decomposed in two separate motions with two different characteristic times. The first motion is the rotation of the rigid curve around the $\mathrm{Z}$ axis. It has short characteristic time $2 \pi / \omega$, where $\omega$ is the angular velocity. In this time one loop of the yarn is unwound. The second motion corresponds to the timevarying form of the balloon. It has long characteristic time of the order of the time in which one layer of the yarn is unwound from the package.

Such a decomposition makes sense only for packages (or layers) where the number of loops in a layer is high, i.e. for precision-wound packages. Let one layer have 100 loops of yarn. Then both characteristic times differ by two orders of magnitude and it is beneficial to take this decomposition explicitly into account in our equation of motion.

Assuming negligibly small yarn cross-section and ideal elasticity one may derive the following equation of motion in an inertial reference frame (Praček, 2002):

$$
\rho D^{2} \mathbf{r}=\frac{\partial}{\partial S}(T \mathbf{t})+\mathbf{f}_{\mathbf{e}}
$$

Here $\rho$ is the linear yarn mass density, i.e., the mass of the yarn per unit length which is not necessarily constant in an extensible yarn. The vector $\mathbf{r}(s, t)$ is the radius vector pointing to an infinitesimal section of the yarn which at time $t$ is locate at the arc-length s away from the origin. 
The mechanical tension is denoted by $T(s, t)$, and $\mathbf{t}(s, t)$ is the tangential vector to the yarn at the given point; it equals the partial derivative of the radius vector $\mathbf{r}$ with respect to the arc-length $s$, that is $\mathbf{t}=\partial \mathbf{r} / \partial \mathrm{s}$. The vector $\mathbf{f}_{\mathrm{e}}$ denotes the linear density of the external forces which are applied to the segment of yarn under discussion. The operator $D$ is the differential operator of the total time derivative following the motion of a point on the yarn. The unwinding speed, i.e., the speed with which the yarn is being withdrawn through the eyelet, is denoted by $V$.

$$
D=\frac{d}{d t}=\left(\frac{\partial}{\partial t}-V \frac{\partial}{\partial s}\right)
$$

In a rotating reference frame the equation (8) takes a form similar to that of equation (7) (Praček, 2002):

$$
\rho \mathcal{D}^{2} \mathbf{r}=\frac{\partial}{\partial s}(T \mathbf{t})+\mathbf{f}_{\mathbf{e}}-2 \rho \boldsymbol{\omega} \times \mathcal{D} \mathbf{r}-\rho \boldsymbol{\omega} \times(\boldsymbol{\omega} \times \mathbf{r})-\rho \dot{\Phi} \times \mathbf{r},
$$

The differential operator $\mathcal{D}$ which follows the motion of a point on the yarn in the rotating reference frame:

$$
\mathcal{D}=\left(\left.\frac{\partial}{\partial t}\right|_{K^{\prime}}-V \frac{\partial}{\partial s}\right)
$$

is related to the operator $D$ by

$$
D=\mathcal{D}+\omega \times .
$$

This expression is similar to the equation $(\partial / \partial t)_{K}=(\partial / \partial t)_{K}+\omega \mathrm{x}$, which was derived in the third section of the paper. Here, again, the expression becomes meaningful only when applied to some vectorial function $\mathbf{p}$, so that $D \mathbf{p}=\mathcal{D} \mathbf{p}+\boldsymbol{\omega} \times \mathbf{p}$. The analogy with the Newton's second law for a point particle in a rotating reference frame (7) is manifest. Both equations are similar in their structure and the respective terms may be interpreted in the same way. The $\mathcal{D}^{2} \mathrm{r}$ term in the equation of motion can be interpreted as the acceleration of a point in the rotating coordinate system. The other three terms on the left hand can be moved to the right side of the equation and reinterpreted as inertial forces that appear due to the non-inertial character of this observation frame. These are not $»$ real « physical forces, but rather forces that an observer in a non-inertial frame would feel because of inertial effects. To emphasize the difference the inertial forces are also called system forces, virtual forces or pseudo-forces. It should be kept in mind that these forces do not appear in equations of motion if they are written in an inertial frame, even if the motion of the body itself is accelerated. They only appear when the equations are expressed in the form appropriate for a non-inertial observation system. The three inertial forces that we're dealing with are: 
1. $-2 \rho \boldsymbol{\omega} \times \mathcal{D} \mathbf{r}$ the Coriolis force

2. $-\rho \omega x(\omega x \quad$ the centrifugal force

3. $-\rho \omega \times \mathbf{r}$ and the Euler force due to angular acceleration

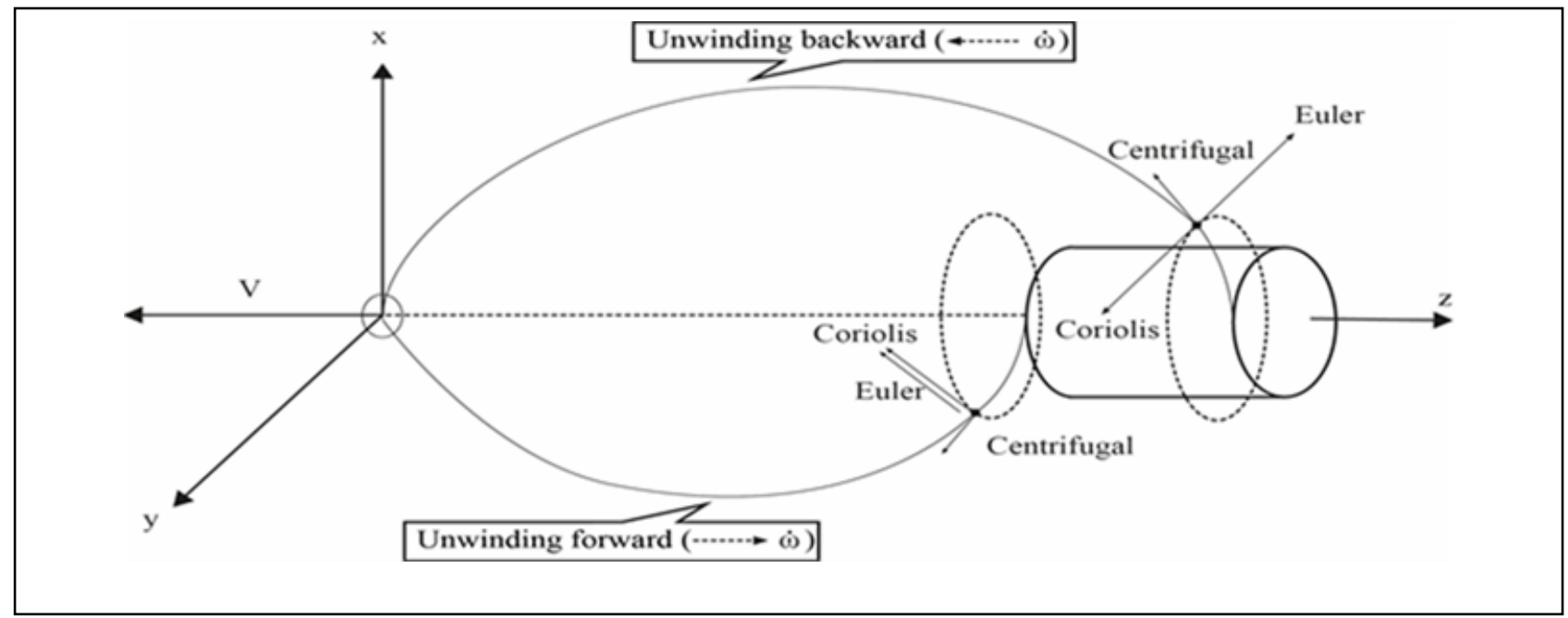

Fig. 2. Inertial forces on a short yarn segment during the unwinding

In most of the introductory textbooks on mechanics the only case that is considered is that in which the angular velocity is constant, so that only Coriolis and the centrifugal forces appear. For this reason the third force is less known and unfortunately it is often neglected even when it plays some role. We were unable to find any mention of this inertial force in the available literature on yarn unwinding and the balloon theory.

It is interesting to describe how an observer standing on a merry-go-round would feel each of these forces. Usually we first notice the centrifugal force; this force »tries « to »eject « us from the merry-go-round. Coriolis force can be seen at work when we throw an object in the radial direction. As seen from our point of view, the object work fly in a straight line as in an inertial frame, but it will deviate in a direction that is perpendicular to its velocity.

The third force could be felt if the merry-go-round would suddenly come to a halt. As our experience tells us, we would most likely fall in this event. This force therefore isn't always negligible: it has very sensible effects when the angular velocity suddenly changes. We will now show when this force should be taken into account in the balloon theory.

On cylindrical packages the angular velocity depends on the unwinding speed V, the package radius $\mathrm{c}$ and on the winding angle $\phi$ (Praček, 2010; Praček, 2010; Praček et al., 2011: Praček et al., 2011).

$$
\omega=\frac{V \cos \phi}{c(1-\sin \phi)}
$$


The unwinding speed and the package radius are approximately constant in the time interval required to unwind a few layers of yarn.

On the other hand, the winding angle $\phi$ is different in each layer of a crosswound package: it is approximately constant when the unwinding point is in the middle of the package and it changes sign near the edges of the package. Variations of $\phi$ lead to sudden changes of angular velocity near the edges.

We have performed numerical simulations of unwinding from both cylindrical and conic cross-wound packages. We show the time dependence of the position of the radius of the package at the unwinding point $\mathrm{c}(\mathrm{t})$, the winding angle $\phi(\mathrm{t})$ and the dimensionless angular velocity $\Omega(\mathrm{t})=\mathrm{c} \omega / \mathrm{V}$ for a cylindrical (full line) and a conic package (dashed line), see Fig. 3.

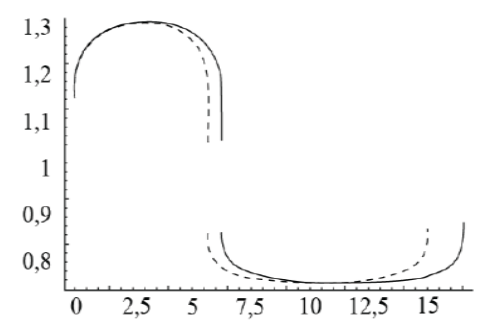

(d) $\Omega(\mathrm{t})$

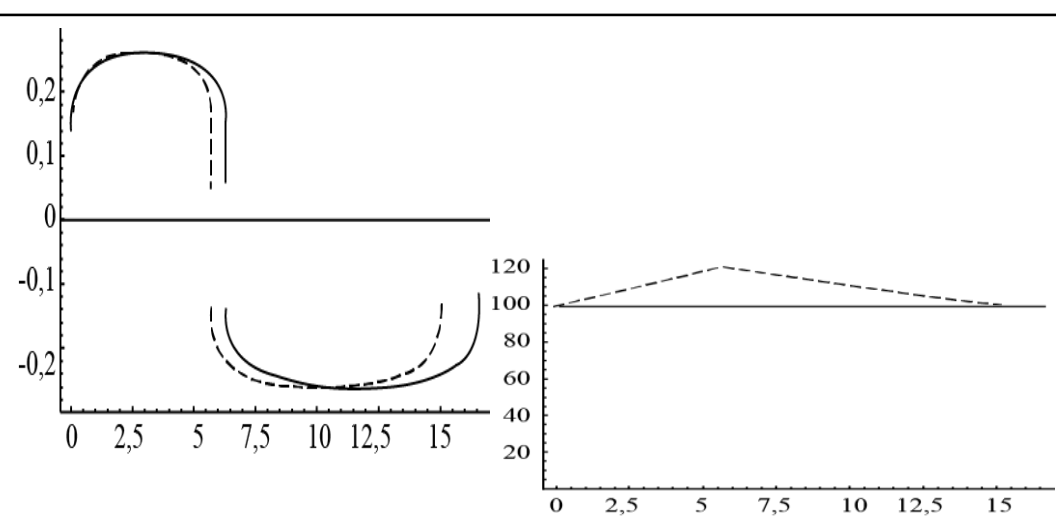

(c) $\varphi(\mathrm{t})(\mathrm{rad})$

(b) $\mathrm{c}(\mathrm{t}) \mathrm{mm}$

Fig. 3. Results of simulation of unwinding from cylindrical and conic packages.

We indeed see that when the unwinding point is in the middle of the package, the conditions are changing slowly with time. The quasi-stationary approximation can then be applied, as is done in most of the theoretical works devoted to yarn unwinding. The time dependence is shifted to the boundary conditions while the equation of motion is simplified to:

$$
\frac{\partial^{2} \mathbf{r}}{\partial s^{2}}-2 \Omega \times \frac{\partial \mathbf{r}}{\partial s}+\Omega \times(\Omega \times \mathbf{r})=\frac{\partial}{\partial s}\left(T \frac{\partial \mathbf{r}}{\partial s}\right)+\mathbf{f} .
$$

Near the front and read end of the package the conditions quickly change from two different quasi-stationary regimes. The third non-inertial force can then become a very large quantity. In the figure 2 we show directions of inertial forces at both edges of a cylindrical package.

\section{Conclusion}

We have shown crucial steps in the derivation of the equation of motion of yarn: the introduction of the non-uniformly rotating observation frame, the calculation of velocity and acceleration and the application of Newton's second law to an infinitesimal segment of yarn. 
The angular velocity $\omega$ is larger during the unwinding in the backward direction as during the unwinding in the forward direction, which is a consequence of a finite winding angle.

The difference is very small in densely wound packages, while it may become substantial in cross-wound packages. The importance of the Euler may be estimated if it is compared with the centrifugal force. The ratio is

$$
\frac{m \dot{d} \dot{r}^{\prime}}{m \omega^{2} r^{\prime}}=\frac{\ddot{X}}{\omega^{2}}=\frac{2 \pi}{n} \frac{\omega_{+}-\omega_{-}}{\omega_{0}}
$$

The factor $2 \pi / \mathrm{n}$ may be taken to be approximately equal to 1 , since $n$ is some small number. Our future research will be based to the ration between the difference of the angular velocity for unwinding in either direction and their average. In crosswound packages the difference between the two angular velocities may be up to a few ten percent, therefore the Euler's force is sizable.

\section{References}

Barr, A. E. D., Catling, H. (1976). Manual of Cotton Spimmimg, Volume Five. Butterworth

Fraser, W. B., Ghosh, T. K., Batra, S. K. (1992). On unwinding yarn from cylindrical package. Proc. R. Soc. Lond. A, 436 479-498.

Fraser, W. B., (1992). The effect of yarn elasticity on an unwinding ballon. J. Tex. Inst, 83 603-613.

Giancoli DC. (2009) Physics for scientists \& engineers : With modern physics. 4th ed. Upper saddle River, NJ: Pearson Education International.

Goldstein H, Poole C and Safko J (2002). Classical mechanics. 3rd ed. San Francisco, CA; Addison Wesley/Upper Saddle River, NJ: Person Education International.

J.D.Clark, W.B.Fraser, R. Sharma and C.D.Rahn. (1998). The dynamic response of a ballooning yarn:theory and experiment. Proc. R. Soc. Lond. A, 454 2767-2789.

Kothari,V.K.,Leaf G.A.V. (1979). The unwinding of yarns from packages, PartII: The theory of yarn-unwinding.J.Text.Inst 70 (3)95-105.

Mack, C. (1953). Theoretical study of ring and cap spinning balloon curves(with and without air drag).J.Text.Inst 44 483-498.

Padfield, D. G. (1956). The Motion and Tension of an Unwinding Thread. Proc. R. Soc., vol. A245, 382-407.

Praček, S. (2002). Modification of yarn unwinding dynamics. Dissertation, University of Ljubljana

Praček, S. (2010). Theoretical model of unwinding process from packages, Annals of DAAAM for 2010 \& Proceedings of the 21 st International DAAAM Symposium, 20-23rd October 2010, Zadar, Croatia, ISSN 1726-9679, ISBN 978-3-901509-73-5, 
Katalinic, B. (Ed), pp. 1469-1470, Published by DAAAM International Vienna, Vienna

Praček, S. (2010). High-speed yarn transport systems simulation, Annals of DAAAM for 2010 \& Proceedings of the 21 st International DAAAM Symposium, 20-23rd October 2010, Zadar, Croatia, ISSN 1726-9679, ISBN 978-3-901509-73-5, Katalinic, B. (Ed), pp. 1471-1472, Published by DAAAM International Vienna, Vienna Praček, S. Sluga, F. Možina, K. Franken, G. (2011). Cylindrical packages simulations, Annals of DAAAM for 2011 \& Proceedings of the 22 st International DAAAM Symposium, 23-26th November 2011, Vienna, Austria, ISSN 1726-9679, ISBN 978-3-901509-83-4, Katalinic, B. (Ed), pp. 0919-0920, Published by DAAAM International Vienna, Vienna

Praček, S. Sluga, F. Možina, K. Franken, G. (2011).Conic packages oscillations, Annals of DAAAM for 2011 \& Proceedings of the 22 st International DAAAM Symposium, 23-26th November 2011, Vienna, Austria, ISSN 1726-9679, ISBN 9783-901509-83-4, Katalinic, B. (Ed), pp. 0921-0922, Published by DAAAM International Vienna, Vienna 